Annals of Pure and Applied Mathematics

Vol.20, No.1, 2019, 13-19

ISSN: 2279-087X (P), 2279-0888(online)

Published on 17 July 2019

Annals of

www.researchmathsci.org

DOI: http://dx.doi.org/10.22457/apam.624v20n1a3

Pure and Applied

Mathematics

\title{
On Distinct Integers the Sum of Whose Reciprocals \\ Equals 1, 2, 3 \\ Nechemia Burshtein
}

\section{Arlozorov Street, Tel - Aviv 6209814, Israel}

Email: anb17@netvision.net.il

Received 2 July 2019; accepted 16 July 2019

Abstract. In this article, we discuss the diophantine equation $\sum_{i=1}^{k} \frac{1}{X_{i}}=1$ in distinct positive integers. Old and new solutions with odd and even integers are exhibited, and related problems are mentioned. In this context, we investigate the equation $\sum_{i=1}^{k} \frac{1}{X_{i}}=2$. We construct and demonstrate solutions with odd and even integers, and also with even integers only. We also consider the equation $\sum_{i=1}^{k} \frac{1}{X_{i}}=3$, and exhibit a solution with odd and even integers. All the solutions are derived without the aid of a computer.

Keywords: Diophantine equations, Unit fractions

AMS Mathematics Subject Classification (2010): 11D68, 11D72

\section{On distinct unit fractions whose sum equals 1}

Hereafter, all mentioned integers are positive integers. A fraction $\frac{a}{b}$ whose numerator is $a=1$ is called a unit fraction (in short uf).

The Diophantine equation in integers

$$
\frac{1}{x_{1}}+\frac{1}{x_{2}}+\cdots+\frac{1}{x_{k}}=1 \quad 1<x_{1}<x_{2}<\cdots<x_{k}
$$

has often been mentioned in the literature. Many authors have considered equation (1). Among them are the late Erdős, Graham, Sierpiński, Barbeau, Johnson, Burshtein and others. Many questions revolve about equation (1). Questions such as: does (1) have a solution for every $x_{1}$, a solution with odd $x_{i}$ only, with even $x_{i}$ only, or when $x_{i} \nmid x_{j}$ for $i \neq j$, and also other ones. We mention that (1) is connected to the topic of Covering Systems, and analogous questions have been asked by Erdős on the moduli of Covering Systems.

From [11] it is known that (1) has a solution for each value $x_{1}$. In [5], Burshtein proved that exactly five solutions $\mathbf{B}_{\mathbf{1}}-\mathbf{B}_{\mathbf{5}}$ of (1) exist when $x_{1}=3$, all $x_{i}$ are odd and $k=9$. Sierpiński [16] exhibited a solution with $x_{1}=3, x_{i} \in 3^{\alpha} 5^{\beta} 7^{\gamma}, k=11$ and $x_{11}=$ 945. Burshtein [8] improved this solution by providing a solution of the same nature, 
Nechemia Burshtein

but with $x_{11}=135$. He also showed [4] when $x_{i} \in 3^{\alpha} 5^{\beta} 7^{\gamma}$ that (1) has exactly 17 solutions. Two solutions with $x_{1}=5$, all $x_{i}$ are odd and $k=21,23$ are demonstrated in [7]. Burshtein [9] and Graham [12] independently raised the question if (1) has a solution when $x_{i} \nmid x_{j}$ for $i \neq j$. Burshtein [8], the first to provide such a solution to (1), received a reward of $\$ 10$ offered by Erdős [9]. More such solutions were later obtained by Barbeau, Burshtein and Johnson in $[1,2,3,6,7,15]$. The results of Burshtein in [3, 6, 8] are also cited in [13].

In our discussion, we shall use the identity

$$
\frac{1}{n}=\frac{1}{n+m}+\frac{m}{n(n+m)}, \quad n \geq 1, \quad m \geq 1 .
$$

The simplest and most trivial solution of (1) is

$$
\frac{1}{2}+\frac{1}{3}+\frac{1}{6}=1
$$

By (2), $\frac{1}{3}=\frac{1}{4}+\frac{1}{12}$, and hence the set $E=\{2,4,6,12\}$ is the smallest possible set of even integers $x_{i}$ when $x_{1}=2$. Another solution of (1) with $x_{1}=4, x_{i}$ are even, $k=14$ and $\sum_{i=1}^{14} \frac{1}{X_{i}}=1$ is given by the set $S$.

$$
S=\{4,6,8,10,12,16,20,24,30,40,48,50,60,200\} .
$$

The set $S$ is constructed as follows. Consider the three sets $U_{1}, U_{2}, U_{3}$ :

$$
U_{1}=\{1\}, \quad U_{2}=\{2,3,6\}, \quad U_{3}=\{4,5,20\},
$$

where $\frac{1}{4}+\frac{1}{5}+\frac{1}{20}=\frac{1}{2}$. Then

$\frac{1}{2}\left(\frac{1}{2}+\frac{1}{3}+\frac{1}{6}\right)=\frac{1}{4}+\frac{1}{6}+\frac{1}{12}=\frac{1}{2}$

3 uf.

$\frac{1}{8}\left(1+\left(\frac{1}{2}+\frac{1}{3}+\frac{1}{6}\right)\right)=\frac{1}{8}+\frac{1}{16}+\frac{1}{24}+\frac{1}{48}=\frac{2}{8}=\frac{1}{4}, \quad 4$ uf.

$\frac{1}{10}\left(1+\left(\frac{1}{2}+\frac{1}{3}+\frac{1}{6}\right)+\left(\frac{1}{4}+\frac{1}{5}+\frac{1}{20}\right)\right)=\frac{1}{10}+\frac{1}{20}+\frac{1}{30}+\frac{1}{40}+\frac{1}{50}+\frac{1}{60}+\frac{1}{200}=\frac{2.5}{10}=\frac{1}{4}, \quad 7 \mathrm{uf}$.

The 14 even integers in $S$ have been determined, and the sum of their reciprocals is 1 . The LCM of the integers in $S$ is $\operatorname{LCM}(S)=2^{4} \cdot 3 \cdot 5^{2}$. Hence, when $x_{1}=4$, the values $k=14$ and $x_{14}=200$ are the smallest best possible values, and are attained in $S$. The sets $E$ and $S$ were cited in [7].

\section{On distinct unit fractions whose sum equals 2}


On Distinct Integers the Sum of Whose Reciprocals Equals 1, 2, 3

In this section, we consider solutions to the equation $\sum_{i=1}^{k} \frac{1}{X_{i}}=2$. For a set which consists of odd and even integers, we demonstrate solutions when $x_{1}=2$ and when $x_{1}=$ 3 . For a set consisting of even integers only, a solution with $x_{1}=2$ is exhibited.

Theorem 2.1. If $x_{1}=2$, then there exist at least two sets $K, N$ of odd and even integers such that $k=12$ and $\sum_{i=1}^{12} \frac{1}{X i}=2$.

Proof: Consider the following six sums

$\frac{1}{2}+\frac{1}{3}+\frac{1}{6}=1, \quad \frac{1}{4}+\frac{1}{5}+\frac{1}{20}=\frac{1}{2}, \quad \frac{1}{7}+\frac{1}{42}=\frac{1}{6}, \quad \frac{1}{8}+\frac{1}{24}=\frac{1}{6}, \quad \frac{1}{9}+\frac{1}{18}=\frac{1}{6}, \quad \frac{1}{10}+\frac{1}{15}=\frac{1}{6}$

obtained by using identity (2).

The above odd and even denominators then yield the two sets of integers $K, N$

$$
\begin{gathered}
K=\{2,3,4,5,6,8,9,10,15,18,20,24\}, \\
N=\{2,3,4,5,6,7,8,9,18,20,24,42\},
\end{gathered}
$$

where $x_{1}=2, k=12, \sum_{i=1}^{12} \frac{1}{X i}=2, \operatorname{LCM}(K)=2^{3} \cdot 3^{2} \cdot 5=360, \operatorname{LCM}(N)=2^{3} \cdot 3^{2}$. $5 \cdot 7=2520$.

This completes our proof.

Remark 2.1. The set $N$ clearly implies that the sum 2 cannot be achieved with 9 uf composed of the first 9 consecutive integers $x_{i}$. Therefore, in any solution of $\sum_{i=1}^{k} \frac{1}{X_{i}}=$ 2 it follows that $k>9$. When $x_{1}=2$, and without a formal proof, we may presume that $\sum_{i=1}^{11} \frac{1}{X_{i}} \neq 2$. If our presumption is indeed true, it will then follow that $k=12$ is the minimal value for $\sum_{i=1}^{k} \frac{1}{X_{i}}=2$ as in $K, N$. Moreover, one could easily verify that the sum 2 can be obtained only when the number $M(p)$ of distinct primes in $\operatorname{LCM}(V)$ of any set $V$ satisfies $M(p) \geq 3$. The minimal value $M(p)=3$ is actually attained in $\operatorname{LCM}(K)$ of the set $K$.

Theorem 2.2. If $x_{1}=3$, then there exist at least five sets of odd and even integers such that $k=23$ and $\sum_{i=1}^{23} \frac{1}{X i}=2$.

Proof: The construction of the first such set $H$ is as follows. Consider the two sets

$\mathbf{B}_{1}=\{3,5,7,9,11,15,21,231,315\}, \quad k=9, \quad \sum_{i=1}^{9} \frac{1}{X_{i}}=1$,

and the previously mentioned set $S$ 


\section{Nechemia Burshtein}

$S=\{4,6,8,10,12,16,20,24,30,40,48,50,60,200\}, \quad k=14, \quad \sum_{i=1}^{14} \frac{1}{X_{i}}=1$.

The two sets differ in their nature. The first set consists of only odd integers, whereas the second set consists of even integers only. Therefore, the combined set $H$ of $\mathbf{B}_{1}$ and $S$ whose integers are arranged in ascending order

$H=\{3,4,5,6,7,8,9,10,11,12,15,16,20,21,24,30,40,48,50,60,200,231,315\}$

satisfies the conditions $x_{1}=3, k=23$ and $\sum_{i=1}^{23} \frac{1}{X_{i}}=2$.

Four more distinct sets $\mathbf{B}_{2}-\mathbf{B}_{5}$ exist, each of which is composed of odd integers only, where $x_{1}=3, k=9$ and $\sum_{i=1}^{9} \frac{1}{X_{i}}=1$. Hence, the union of each of the sets $\mathbf{B}_{\mathbf{2}}-\mathbf{B}_{\mathbf{5}}$ with the set $S$ yields a set of the same nature as $H$, in which $x_{1}=3, k=23$ and $\sum_{i=1}^{23} \frac{1}{X_{i}}=2$.

The assertion then follows.

Theorem 2.3. If $x_{1}=2$, then there exists a set $T$ all of whose integers are even, $k=46$ and $\sum_{i=1}^{46} \frac{1}{X_{i}}=2$.

Proof: We mention again the former two sets $S$ and $\mathbf{B}_{\mathbf{1}}$.

The set $S$ of even integers is

$S=\{4,6,8,10,12,16,20,24,30,40,48,50,60,200\}, k=14, \quad \sum_{i=1}^{14} \frac{1}{X_{i}}=1$.

In the set

$$
\mathbf{B}_{1}=\{3,5,7,9,11,15,21,231,315\}, \quad k=9, \quad \sum_{i=1}^{9} \frac{1}{X_{i}}=1,
$$

we multiply each $x_{i}$ by 2 . Then $y_{i}=2 x_{i}$, and we obtain a new set $Q$ of even integers

$Q=\{6,10,14,18,22,30,42,462,630\}, \quad k=9, \quad \sum_{i=1}^{9} \frac{1}{y_{i}}=\frac{1}{2}$.

The two sets $Q, S$ have three overlaps, namely the integers $6,10,30$. The sum $\frac{1}{6}+\frac{1}{10}+\frac{1}{30}=\frac{3}{10}$. Using the primes $13,17,19$, and utilizing identity (2), we construct the following three sets $M_{1}, M_{2}, M_{3}$ of even integers, each of which occurs once and only once.

The respective three sets $M_{1}, M_{2}, M_{3}$ are:

$M_{1}=\{26,28,66,130,364,4290\}$

$k=6, \quad \sum_{i=1}^{6} \frac{1}{X_{i}}=\frac{1}{10}$. 
On Distinct Integers the Sum of Whose Reciprocals Equals 1, 2, 3

$M_{2}=\{34,36,44,86,176,612,1496,2992,7310\}, \quad k=9, \quad \sum_{i=1}^{9} \frac{1}{X_{i}}=\frac{1}{10}$.

$M_{3}=\{32,38,76,96,98,152,608,3724,9120,18620\}, k=10, \quad \sum_{i=1}^{10} \frac{1}{X_{i}}=\frac{1}{10}$.

Omitting the three integers $6,10,30$ from the set $S$, and instead inserting the 25 integers in $M_{1}, M_{2}, M_{3}$ yields a new set $G$ of 36 even integers, the sum of whose reciprocals equals 1 .

$G=\{4,8,12,16,20,24,26,28,32,34,36,38,40,44,48,50,60,66,76$, $86,96,98,130,152,176,200,364,608,612,1496,2992,3724,4290$, $7310,9120,18620\}$.

The set $T$ consists of: the integer 2, the 36 integers in $G$, and the 9 integers in $Q$. The 46 integers sequenced in ascending order are distinct, even, and the sum of their reciprocals is equal to 2 .

$T=\{2,4,6,8,10,12,14,16,18,20,22,24,26,28,30,32,34,36,38,40$, $42,44,48,50,60,66,76,86,96,98,130,152,176,200,364,462,608$, $612,630,1496,2992,3724,4290,7310,9120,18620\}$.

The set was achieved without the use of a computer.

This concludes the proof of Theorem 2.3.

\section{On distinct unit fractions whose sum equals 3}

In Theorem 3.1, we demonstrate a set of distinct integers the sum of whose reciprocals is equal to 3 .

Theorem 3.1. If $x_{1}=2$, then there exists a set $D$ of odd and even integers such that $k$ $=69$ and $\sum_{i=1}^{69} \frac{1}{X i}=3$.

Proof: We shall demonstrate three sets $A, B, C$ all of whose integers are distinct. The union of $A, B, C$ is the set $D$ of 69 integers the sum of whose reciprocals equals 3 .

Since $\frac{1}{8}=\frac{1}{12}+\frac{1}{30}+\frac{1}{120}$, it follows that

$$
\frac{1}{2}+\frac{1}{4}+\frac{2}{8}=\frac{1}{2}+\frac{1}{4}+\frac{1}{8}+\left(\frac{1}{12}+\frac{1}{30}+\frac{1}{120}\right)=1 \text {. }
$$

Hence, the set $A$ is

$$
A=\{2,4,8,12,30,120\}, \quad \sum_{i=1}^{6} \frac{1}{X_{i}}=1 .
$$

Without the aid of a computer, Burshtein [2] exhibited a solution of $\sum_{i=1}^{52} \frac{1}{X_{i}}=1$ in which $x_{i}$ are distinct, and $x_{i} \nmid x_{j}$ for $i \neq j$. This solution is the following set $B$. 
$B=\{6,10,14,15,21,22,26,33,34,35,38,39,46,51,55,57,58,62,65$, $69,77,82,87,91,93,95,106,119,122,123,133,155,159,161,183$, $187,202,203,213,265,287,299,319,355,453,497,505,583,671$, 1057, 1313, 1963\}, $\quad \sum_{i=1}^{52} \frac{1}{X_{i}}=1$.

The set $\mathbf{B}_{1}=\{3,5,7,9,11,15,21,231,315\}$, and the set $B$ have two overlaps, the two integers 15, 21. From (2) we have

$\frac{1}{15}=\frac{1}{16}+\frac{1}{15 \cdot 16}, \quad \frac{1}{21}=\frac{1}{24}+\frac{3}{21 \cdot 24}=\frac{1}{24}+\frac{1}{7 \cdot 24}$.

Substituting for 15, 21 in $\mathbf{B}_{1}$ the above four integers 16, 240, 24, 168 yields the set $C$

$C=\{3,5,7,9,11,16,24,168,231,240,315\}$

$$
\sum_{i=1}^{11} \frac{1}{X_{i}}=1
$$

The 69 distinct integers in set $D$ appear in ascending order, and are the union of the 69 integers in $A, B, C$.

$D=\{2,3,4,5,6,7,8,9,10,11,12,14,15,16,21,22,24,26,30,33,34$, $35,38,39,46,51,55,57,58,62,65.69,77,82,87,91,93,95,106,119$, $120,122,123,133,155,159,161,168,183,187,202,203,213,231,240$, $265,287,299,315,319,355,453,497,505,583,671,1057,1313,1963\}$,

$$
\sum_{i=1}^{69} \frac{1}{X_{i}}=3 .
$$

The proof of Theorem 3.1 is complete.

Final remark. Suppose that $1<x_{1}<x_{2}<\cdots<x_{k}$ are integers and $N>3$ is a fixed integer. Then, a sufficient condition for a solution of $\sum_{i=1}^{k} \frac{1}{x_{i}}=N$ is that there exist $N$ sets each of which consists of distinct integers where the sum of the reciprocals of each such set is equal to 1 , and the existing overlaps between the sets are corrected by using identity (2).

\section{REFERENCES}

1. E. J. Barbeau, Expressing one as a sum of distinct reciprocals: comments and bibliography, Eureka (Ottawa), 3 (1977) $178-181$.

2. N. Burshtein, The solution of the equation $\sum_{i=1}^{52} \frac{1}{X_{i}}=1$ in distinct integers each a product of two distinct primes is minimal in every parameter and unique, Journal for Algebra and NumbercTheory Academia, 3 (4) (2013) 209 - 220.

3. N. Burshtein, An improved solution of $\sum_{i=1}^{k} \frac{1}{X_{i}}=1$ in distinct integers when $x_{i} \nmid x_{j}$ for $i \neq j$, Notes on Number Theory and Discrete Mathematics, 16 (2) (2010) 1 - 4.

4. N. Burshtein, All the solutions of the equation $\sum_{i=1}^{11} \frac{1}{X_{i}}=1$ in distinct integers of the form $x_{i} \in 3^{\alpha} 5^{\beta} 7^{\gamma}$, Discrete Mathematics, 308 (18) (2008) 4286 - 4292.

5. N. Burshtein, The equation $\sum_{i=1}^{9} \frac{1}{X_{i}}=1$ in distinct odd integers has only the five known solutions, Journal of Number Theory, 127 (1) (2007) 136 - 144. 
On Distinct Integers the Sum of Whose Reciprocals Equals 1, 2, 3

6. N. Burshtein, Improving solutions of $\sum_{i=1}^{k} \frac{1}{X_{i}}=1$ with restrictions as required by Barbeau respectively by Johnson, Discrete Mathematics, 306 (13) (2006) 1438 1439.

7. N. Burshtein, On distinct unit fractions whose sum equals 1, Discrete Mathematics, 300 (2005) $213-217$.

8. N. Burshtein, On distinct unit fractions whose sum equals 1, Discrete Mathematics, 5 (3) (1973) $201-206$.

9. N. Burshtein, Oral communication to P. Erdős, Nice, September 1970.

10. L. E. Dickson, History of the theory of numbers, vol. 2 (Chelsea, New York, 1966) pp. $688-691$.

11. P. Erdős, On the solutions in integers of $a / b=x_{1}^{-1}+\cdots+x_{n}^{-1}$, Mat. Lapok, 1 (1950) $192-210$.

12. P. Erdős, Written communication, December 1970.

13. R. L.Graham, Paul Erdős and Egyptian Fractions, in: L. Lováz, L. Ruzsa, V. Sós (Eds.), Erdős Centennial, János Bolyai Mathematical Society, 25 (2013), Springer, chapter 10, pp. $289-309$.

14. R. K. Guy, Unsolved Problems in Number Theory, Third edition, Springer, New York, 2004.

15. A. Wm. Johnson, Letter to the Editor, Crux Mathematicorum (= Eureka (Ottawa)), 4 (1978) 190.

16. W. Sierpiński, Sur les décompositions de nombres rationnels en fractions primaires, Mathesis, 65 (1956) $16-32$. 